\title{
3D Printing: Its Application in Pediatric Dental Practice
}

\section{Rajib Sikdar, Anisha Bag, Supreet Shirolkar*, Khooshbu Gayen, Subir Sarkar and Somen Roychowdhury}

Department of Pedodontics and Preventive Dentistry, Dr. R. Ahmed Dental College and Hospital, Kolkata, India

*Corresponding Author: Supreet Shirolkar, Department of Pedodontics and Preventive Dentistry, Dr. R. Ahmed Dental College and Hospital, Kolkata, India.
Received: December 31, 2021

Published: January 25, 2022

(C) All rights are reserved by Rajib Sikdar., et

al.

\section{Abstract}

Three-dimensional (3D) printing is an additive manufacturing technique that entails depositing many layers of material to make a three-dimensional (3D) object. 3D printers are machines that produce representations of items created with CAD software or scanned with a 3D scanner. Selective laser sintering (SLS), stereolithography, fused deposition modeling, and laminated object creation are justa few of the technologies we can employ to print numerous dental goods. The materials can be used to print individual imprint trays, orthodontic models, gingiva masks, and a variety of prosthetics. Diagnostics, maxillofacial growth evaluation, facial asymmetry, and the fabrication of aligners for children with malalignment are all made possible with 3D printed products in paediatric dentistry.

Keywords: Additive Manufacturing; Bioprinting; Bone Augmentation; Regeneration; Scaffolds

\section{Introduction}

The additive manufacturing process or 3D printing process is the production of any object by adding material layer by layer [1]. There are different additive manufacturing methods present: [1] Stereolithography, [2] Fused deposition modeling, [3] Selective Laser Sintering, [4] Polyjet printing, and Bioprinting [2]. Fused deposition modeling is most commonly used due to its wide availability, reliable printing quality, ease of installation and use, and economic [3]. CAD-CAM technology enables the rapid growth of 3D printing technology in the field of dentistry and medicine. Its faster manufacturing rate, painless digital data workflow, and patient friendly approach going to revolutionize modern-day dentistry [1].

\section{Invention and advancements}

In 1980, Hideo Kodmann of the Nego Industrial Development Research Institute in Japan was the first to introduce the notion of layer-by-layer part manufacturing. By using photosensitive light to cure a photosensitive resin, he was able to produce a three-dimensional structure. Despite having devised the concept for the first time in the world, Hideo Kodmann's patent was lost owing to a lack of funding [4].

Charles Hall [1983] invented 3D printing technology, named "Stereolithography". Charles Hull is known as the "Father of 3D Printing." In this process, data is stored in a CAD finalised. STL format and an object were printed. Hall later in 1988 founded the company 3D Systems and introduced the first commercially available 3D printer named SLA-250 [5].

In 1987, Texas University's Carl Decard submitted a patent for Selective Laser Sintering, a new technology. (SLS). DTM licensed the technology, which 3D Systems eventually bought. In the same year, Scott Crump formed Stratasys and submitted a patent for Fused Deposition Modelling (FDM) [4]. 


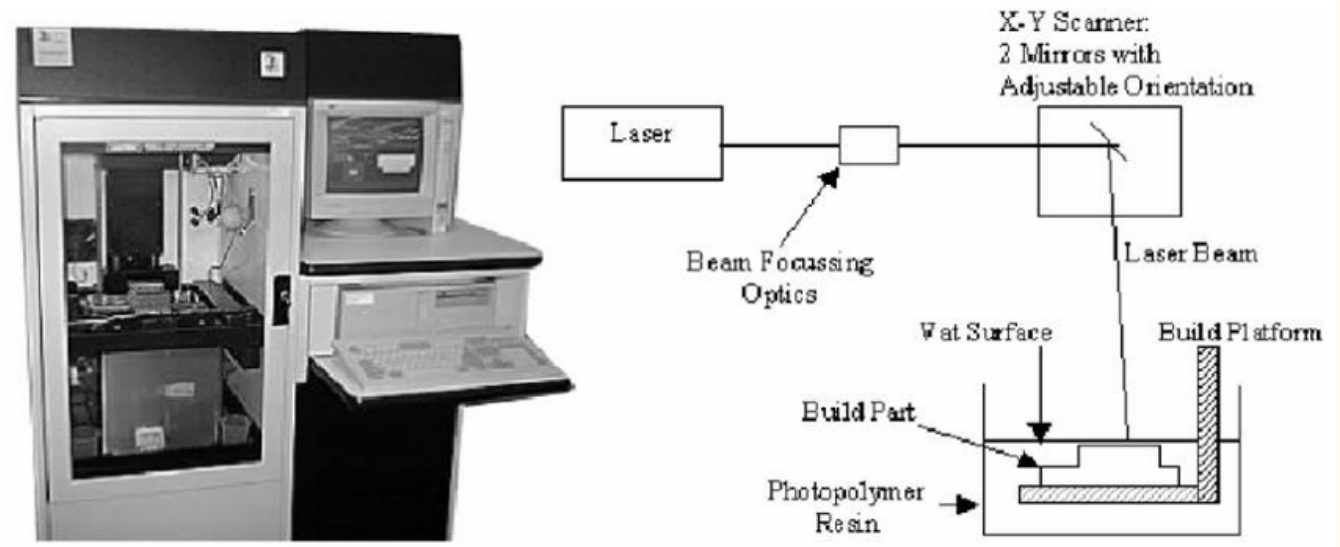

Figure 1: Charles Hall "Stereolithography” 3D printer named SLA-2506.

In 1992, Emanuel Sachs created the phrase "3D Printing." What was once known as additive manufacturing is now known as 3D printing in the realm of Science and technology. Solingen was the first to commercialize Direct Shell Production Casting (DSPC) using ink-jet processes in 1993. In 1995, MIT granted Z corp permission to manufacture starch and plaster models. By the end of the 1990s, only three big businesses remained: 3D Systems, EOS, and Stratasys. They are still at the heart of today's advances in Additive Manufacturing [7].

\section{Process of 3D printing}

The additive manufacturing process can be divided into four

steps. They include $[8,9]$.

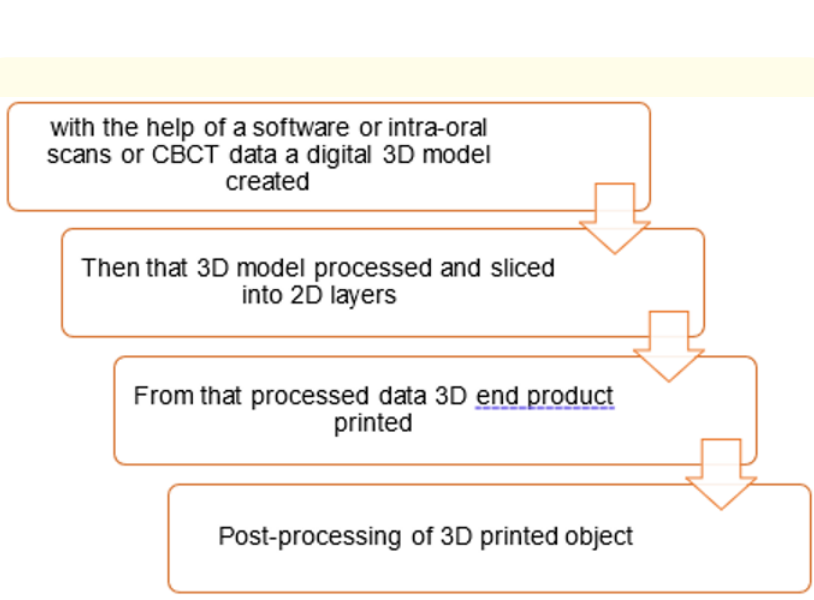

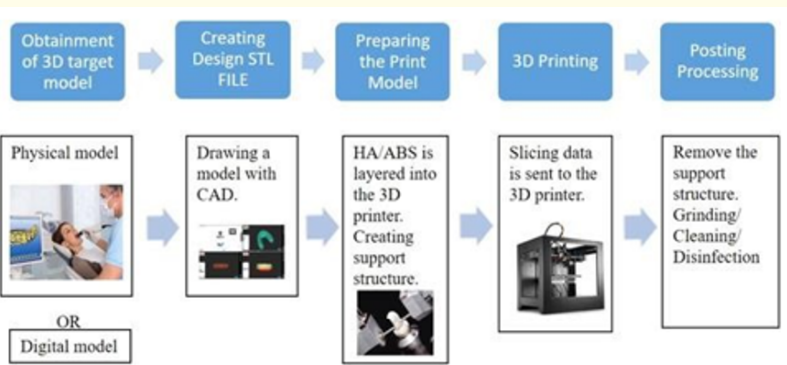

Figure 2: 3D Printing Process [10].

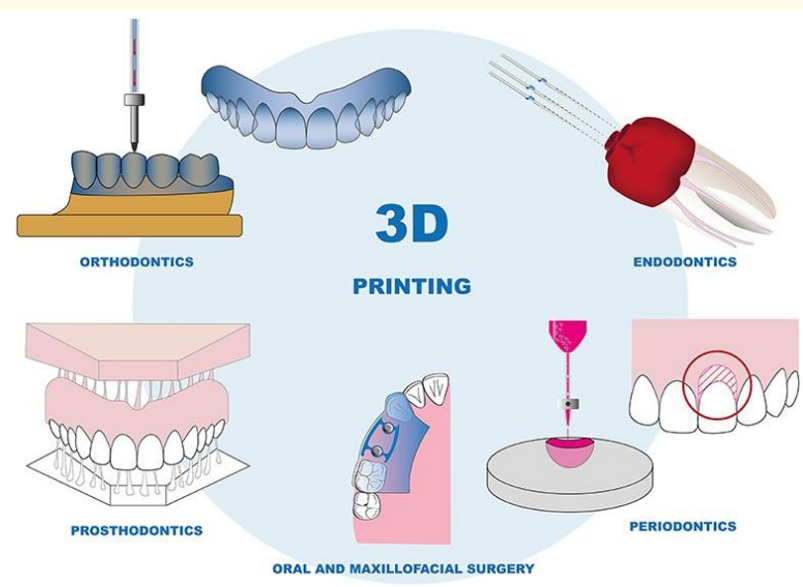

Figure 3: Application of 3D Printing [14]. 


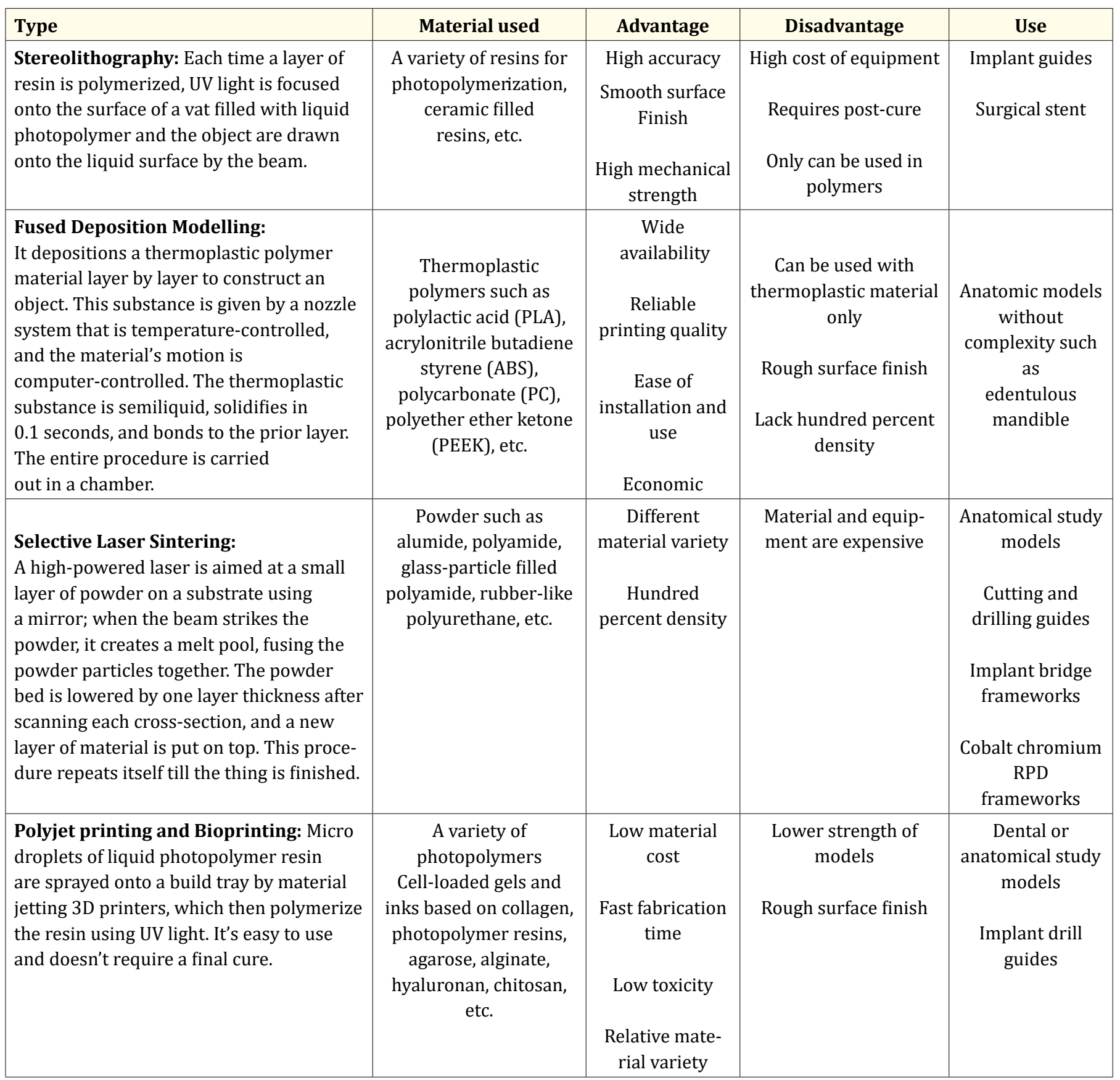

Table 1: Types of 3D Printing Technology [11-13]. 
Surgical pedodontics

In this century of advanced technological engineering, the use of 3D printing in the field of dentistry can lead to accurate diagnosis and successful treatment planning [15]. 3D printed models created with additive manufacturing technology can assist paediatric dentists in visualising accurate anatomy of maxillofacial defects and pre-surgical planning [16]. These accurately built 3D models can act as a reference model during surgery. Klein., et al. (1992) used stereolithography to custom make a model upon which a maxillary prosthesis could be fabricated [17].

The pre-surgical models obtained from CBCT data of the paediatric patients help in the development of surgical guides or surgical stents [16]. These surgical guide scan be very helpful during the planning and placement of implants in patients [18]. Recent developments in bone graft and scaffold printing by 3D printing technology have made this technology one of the most important tools for maxillofacial surgeons. 3D printing technology can develop accurate and three- dimensional scaffolds for orofacial defects and can also be customised for its physical properties like porosity, flexibility, surface texture, hardness, solubility, etc.,according to the needs of that particular treatment modality. Recently, it has also been possible to incorporate osteo-inductive factors like bone morphogenic protein (BMP-2 and BMP-7) and growth factors into the scaffold for better cell adhesion, proliferation, and vascularization, leading to improved tissue regeneration $[19,20]$. These individualized implants and scaffolds for every patient made from 3D printed biocompatible materials help with better tissue regeneration and rehabilitation of orofacial defects $[18,19]$.

Orthognathic surgery of young patients is another field of surgical dentistry where 3D printing technology could be very helpful in the future. Autorotation of the temporomandibular joint during orthognathic surgery leads to condylar instability, which is a major problem. A Personalized orthognathic surgical guide (POSG) system is used to hold the condyle in the correct position with the help of screws and titanium plates [22]. Highly patient-specific titanium plates and screws can be produced by using 3D printing technology with a CAD-CAM device, which results in better surgical compatibility and high accuracy [23].

\section{Management of dental trauma in young and adolescents}

In the future, 3D printed tailored designs and accurate stents in dental traumatology are projected to deliver consistent long-term success.

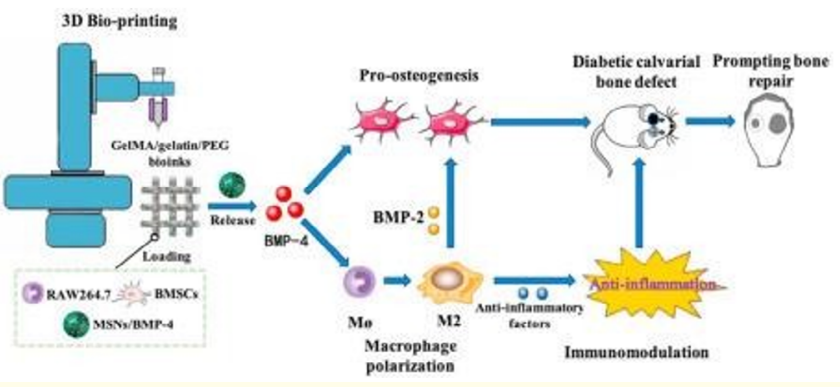

Figure 4: Incorporation of Osteo-inductive Factors [21].

\section{Splint designing}

A trauma splint's goal is to keep injured teeth/teeth in a near natural position while also assisting in the repair of supporting tissues over time. Even with the most advanced adaptations, current splints cannot guarantee proper repositioning and long-term durability.

Any custom-made splint will accomplish two goals

- It will keep the traumatised teeth secure for the required amount of time

- $\quad$ Functioning as a guide to properly reposition them.

When trying surgical relocation of intruded permanent teeth, this second function is critical. The use of digitally scanned information from a cone beam CT scan will enable the construction of such a splint, which may then be cemented orcured into place in the traumatised region [18].

\section{Autotransplantation}

During the early mixed dentition phase, traumatic dental traumas that result in permanent tooth loss preclude implant-based rehabilitation. Auto transplant protocols have recently been developed with a high percentage of success. In tooth autotransplantation, 3D printed templates help to establish a guided atraumatic strategy. This could lead to fewer failures and a better outcome. A new method for tooth autotransplantation using three- dimensional (3D) surgical templates for guided osteotomy preparation and donor tooth insertion was disclosed in the literature.

Vandekar., et al. used 3D printing to scan and reproduce a diseased permanent maxillary central incisor. This quick prototype tooth replica was then used as a guide for determining socket size 
during surgical preparation at the recipient site. This enabled for precise positioning while protecting the periodontal ligament's life [24]. A comparable method can be used with 3D printed surgical guidance for future auto transplant surgery.

\section{Aesthetic restoration of fractured anterior teeth}

3D printed templates are an effective, convenient, and elegant approach for direct resin composite replacement of injured anterior teeth. They aid in the accurate replication of the fractured tooth's architecture, colour, and translucency [25].

\section{Reconstruction of fractured mandible}

After treatment of complex mandibular fractures, contouring of bone plates is a time-consuming and labor-intensive technique. Preoperative planning for difficult mandibular fracture repair and plate contouring can be made easier with 3-D-printed templates [26].

3D printers can produce customised bone tissue for patients, as well as biomimetic scaffolds for bone cell augmentation and tissue development and differentiation. Novel In addition to bone regeneration, 3D printed alginate-peptide hybrid scaffolds can be used. Alginate-based scaffolds are said to provide a stable environment for stem cell proliferation, according to studies. We can print scaffolds out of composite powders. Calcium phosphate $\left(\mathrm{Ca}_{3} \mathrm{PO}_{4}\right)_{2}$ powders can be blended with a calcium sulphate $\left(\mathrm{CaSO}_{4}\right)$-based $3 \mathrm{D}$ printing (3DP) powder to make scaffolds that can also be utilised for bone augmentation [27].

\section{Pediatric rehabilitation}

Rehabilitation should be the main focus of this century. But this rehabilitation process sometimes becomes very time-consuming, cumbersome, and physically andpsychologically traumatic for a young patient, a mentally and physically disabled child, or a child with TMJ defects or orofacial deformities $[29,30]$. It may require a multistage process and highly skilled dental technicians to produce a good maxillofacial prosthesis for orofacial defects [29]. With the recent advancement of digital dentistry, intra-oral scanners.

CAD-CAM devices, along with 3D printing technology, can directly print maxillofacial prostheses from the digital data workflow without traumatising young patients or special children. 3D

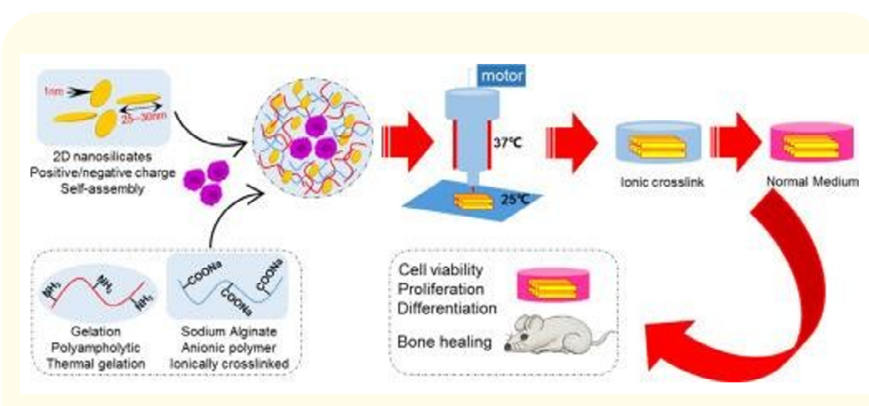

Figure 5: Bone Cell Augmentation [28].

printing technology can minimise this multi-stage process, limit dental appointments, and produce highly accurate prostheses and improve functionality and aesthetics for better psycho-social development of children [31].

Recent studies have shown that ceramic prostheses produced by using the lithography technique have similar physical properties to conventional milled ceramics [27]. Studies have shown that 3D printed metal implant prostheses can be successfully produced by selective laser melting and electron beam melting [32].

3D printed interim resin restorations and metal crowns have an accurate marginal fit andare comparable to conventionally fabricated restorations [33].

\section{Orthodontics for young and adolescents}

In the near future, 3D printing technology could be a revolutionary tool for patient diagnosis, treatment planning, and most importantly, patient motivation. With 3D printing technology and artificial intelligence software, it is now very much possible to virtually present facial changes to patients. The patient can see and feel the three-dimensional models of corrected arches and precise changes in their hand [34].

Stereolithography can 3D print customised braces with specific tip and torque or individualised clear aligners for the patients [35]. A recent study done by Reynolds., et al. on a 3D printed model of the mandible showed results in line with Bjork's study of mandibular growth by implantable marker. This will aid the paediatric surgeons in conducting craniofacial surgery with the help of an orthodontist and 3D printed growth models [36]. 
Additive printing technology can be used in a customised splint for young patients with TMJ dysfunction [37]. In the foreseeable future, it will be very much possible to 3D print precise myofunctional appliances like the Herbst appliance, the Activator appliance, and twin block appliances for growing patients according to their needs. These precise myofunctional appliances can achieve excellent intraoral fit, leading to better patient compliance and treatment results. Other special appliances like sleep apnea appliances, obturators, and feeding appliances for cleft lip and palate patients can also be 3D printed [38]. Bioprinting of complex oral tissue structure scan help to study the biological response to forces induced by orthodontic treatment [39].

\section{Pediatric endodontics}

Sometimes it is impossible to analyse a complex root canal system with obliterated canals and lateral canals using 2D radiographic images. When the root canal system becomes obliterated as a result of rapid dentinogenesis and dystrophic calcification, a custom-designed endodontic guide is created utilising 3D digital planning for predictable navigation of the obliterated pulp. This reduces the risk of severe dentine destruction/root perforation by preventing iatrogenic root damage [40]. So 3D printed models of patients' root canal architecture from digital data from the patient's CBCT scan can be very helpful in treatment planning and access preparation. It can also be used in surgical endodontics such as apicoectomy [41].

Regenerative endodontics will be the focus of this century in the field of endodontics. Additive manufacturing will be used in delivering stem cells, producing biocompatible pulp scaffolds, and carrier membranes for PRP. 3D printing can be used to develop injectable calcium hydroxide molecules, and growth factors and gene therapy in regenerative endodontics [42]. Calcium hydroxide medicament and calcium phosphate cement in the form of porous scaffolds developed by 3D printing technology can be used for regeneration of the pulp-dentin complex [43].

\section{Tissue regeneration and smile designing}

3D printing can mainly be used in gingival aesthetic surgeries. 3D printed individualised surgical guides are used for gingivectomy and smile designing in young adolescent patients [44]. But additive manufacturing technology can revolutionise and shift the focus towards regenerative periodontics. 3D printed scaffolds help in delivering growth factors, genetically modified cells, and bioactive proteins for restoring resorbed periodontal tissue [45]. These 3D printed scaffolds can be used in guided tissue regeneration procedures. It can lead to controlled tissue generation of surrounding periodontal structures in juvenile periodontitis, aggressive periodontitis, or endocrine disorder patients by preventing the ingrowth of rapidly generating tissue such as oral epithelium and allowing slow growth of periodontal tissue [46]. Scaffolds or carrier membranes developed from additive manufacturing technology using 3D polycaprolactone have improved integrity and function and are more resistant to occlusal forces [47].

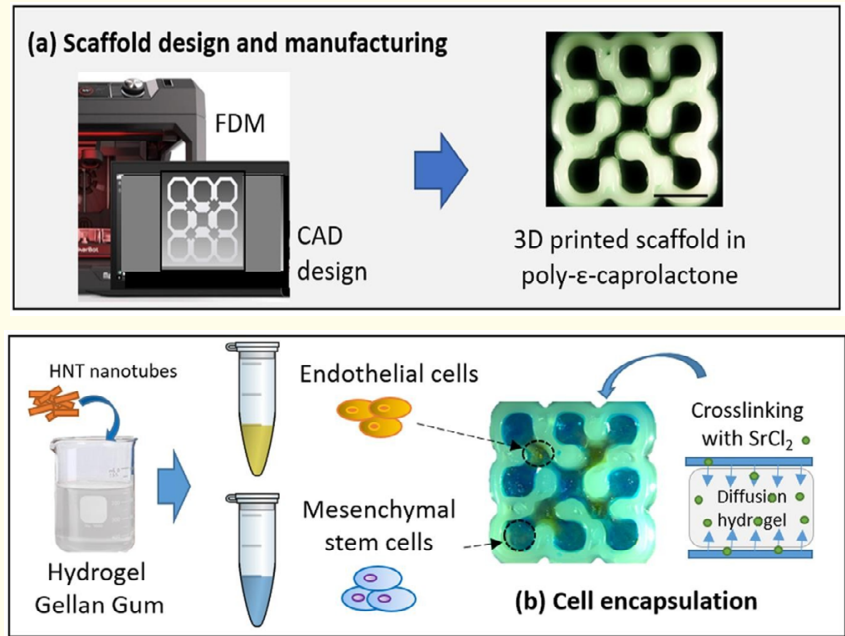

Figure 6: Scaffold Generation [48].

\section{Educational approach}

3D printed structures are a great way to learn about complicated anatomical structures [49]. Students often struggle to properly examine a child patient, leading to pain, discomfort and bleeding during examination. 3D printed models stimulating teeth, periodontal and perioral structures with respective tissue characteristics are very helpful to orient students about proper examination and develop skills and proprioception required for different dental procedures [50]. These three-dimensional anatomical structures give students a detailed and precise spatial view of the complexity of the structure and its pathology and help them plan treatment modalities with great accuracy [51]. 
In complex cases of paediatric rehabilitation such as amelogenesis imperfecta or dental fluorosis, customised 3D-printed patientbased models help dentists or students train for crown and veneer preparation [52]. 3D printed models developed from periodic intra-oral scans are a great way to study the growth pattern of a child. It helps dentists educate patients about their occlusion or habits and motivate them to seek orthodontic correction of malocclusions [53].

Customised real-patient-based 3D printed models with complex root canal architecture help students or young dentists train and develop visual and tactile sensations with a realistic approach and improve their endodontic skills. This 3Dprinted model base training will better prepare them for real-life scenarios and minimise procedural errors $[41,54]$.

\section{Future perspective}

Additive printing technology can revolutionise dentistry. Currently, it is mainly used in surgical planning and indirect implant preparation or aligners for orthodontic correction. As technology advances, creating new heights every day, 3D printing has the capacity to modernise other aspects of dentistry as well. It can be used in the preparation of crowns as newer biocompatible printable materials are developed every day. Ongoing research for 3D printed molecules of intra-canal medicaments with greater healing potential or the discovery of a growth factor delivery system in the periapical region has the potential to transform dentistry.

\section{Conclusion}

3D printing, along with CAD-CAM, intra-oral scanning, and CBCT data, has the ability to revolutionise dentistry. It has the ability to generate sophisticated three- dimensional anatomical structures for educational and training purposes, as well as research and treatment planning. With the help of 3D printed models and surgical guides, complex cranio-facial surgeries have become more predictable, less invasive, and more precise. The lack of qualified operators, greater operating costs, fewer compatible materials, the need for post-processing, regular maintenance requirements, and stringent adherence to health and safety issues are all important downsides of this technology. With the advancement of this technology, within a few decades, 3D printing and digital dentistry are going to significantly change the field of dentistry.
Bibliography

1. Dawood A., et al. "3D printing in dentistry". British Dental Journal 219.11 (2015): 521.

2. Knowlton S., et al. "Bioprinting for cancer research". Trends in Biotechnology 33 (2015): 504-513.

3. Huang Y., et al. "3D bioprinting and the current applications in tissue engineering". Biotechnology Journal12 (2017): 1600734 .

4. The History of 3D Printing: 3D Printing Technologies from the 80 s to Today.

5. Ventola CL. "Medical applications for 3D printing: current and projected uses". Pharmacy and Therapeutics 39.10 (2014): 704.

6. Ebert-Uphoff I., et al. "Rapid Prototyping for Robotics. In M. Kordic, V., Lazinica, A., Merdan, ed. Cutting Edge Robotics”. Mammendorf, Germany: Pro Literatur Verlag (2005): 17-46.

7. Singh R and Chhabra M. "Three-dimensional printing". Reference Module in Materials Science and Materials Engineering.

8. Chia HN and Wu BM. "Recent advances in 3D printing of biomaterials". Journal of Biological Engineering 9 (2015): 4.

9. Ligon SC., et al. "Polymers for 3D printing and customized additive manufacturing". Chemical Reviews 117 (2017): 1021210290.

10. Lin L., et al. "3D Printing and Digital Processing Techniques in Dentistry: A Review of Literature". Advanced Engineering Materials 21.6 (2019): 1801013.

11. Van Noort R. "The future of dental devices is digital". Dental Materials 28.1 (2012): 3-12.

12. Jain R., et al. "Recent Trends of 3-D Printing in Dentistry- A review". Annals of Prosthodontics and Restorative Dentistry 2.4 (2016): 101-104.

13. Azari A and Nikzad S. "The evolution of rapid prototyping in dentistry: a review". Rapid Prototyping Journal 15.3 (2009): 216-225.

14. Oberoi G., et al. "3D Printing-Encompassing the Facets of Dentistry". Frontiers in Bioengineering and Biotechnology 6 (2018): 172. 
15. Marsh JL and Vannier MW. "Surface imaging from computerized tomographic scans". Surgery 94 (1983): 159-165.

16. Erickson DM., et al. "An opinion survey of reported benefits from the use of stereolithographic models". Journal of Oral and Maxillofacial Surgery 57 (1999): 1040-1043.

17. Klein HM., et al. "[Stereolithographic model construction based on 3- dimensional reconstructed CT sectional image sequences]". Rights of First Offer 156 (1992): 429-432.

18. Nayar S., et al. "Rapid prototyping and stereolithography in dentistry". Journal of Pharmacy and Bioallied Sciences 7.1 (2015): S216.

19. Knippenberg M., et al. "Osteogenesis versus chondrogenesis by BMP-2 and BMP-7 in adipose stem cells". Biochemical and Biophysical Research Communications 342 (2006): 902-908.

20. Miller ED., et al. "Inkjet printing of growth factor concentration gradients and combinatorial arrays immobilized on biologically-relevant substrates". Combinatorial Chemistry and High Throughput Screening 12 (2009): 604-618.

21. Sun X., et al. "Three-dimensional bioprinting of multicell-laden scaffolds containing bone morphogenic protein- 4 for promoting M2 macrophage polarization and accelerating bone defect repair in diabetes mellitus". Bioactive Materials 6.3 (2021): 757-769.

22. Li B., et al. "A new approach of splint-less orthognathic surgery using a personalized orthognathic surgical guide system: a preliminary study". International Journal of Oral and Maxillofacial Surgery 46 (2017): 1298-1305.

23. Philippe B. "Custom-made prefabricated titanium miniplates in Le Fort I osteotomies: principles, procedure and clinical insights". International Journal of Oral and Maxillofacial Surgery 42 (2013): 1001-1006.

24. Vandekar M., et al. "Rapid Prototyping as an adjunct for auto transplantation of impacted teeth in the esthetic zone". Journal of Clinical Orthodontics 49 (2015): 711-715.

25. Xia J., et al. "Direct resin composite restoration of maxillary central incisors using a 3D-printed template: two clinical cases". BMC Oral Health 18 (2018): 158.

26. Sinha P., et al. "A 3-dimensional-printed short-segment template prototype for mandibular fracture repair". JAMA Facial Plastic Surgery 20 (2018): 373-380.
27. Uçar Y., et al. "Layered manufacturing of dental ceramics: fracture mechanics, microstructure, and elemental composition of lithography-sintered ceramic". The Journal of Prosthodontics (2018).

28. Liu B., et al. "3D-bioprinted functional and biomimetic hydrogel scaffolds incorporated with nanosilicates to promote bone healing in rat calvarial defect model". Materials Science and Engineering: C 112 (2020): 110905.

29. Yuzbasioglu E., et al. "Comparison of digital and conventional impression techniques: evaluation of patients' perception, treatment comfort, effectiveness and clinical outcomes". BMC Oral Health 14 (2014): 10.

30. Kim JE., et al. "Fabrication of a complete, removable dental prosthesis from a digital intraoral impression for a patient with an excessively tight reconstructed lip after oral cancer treatment: a clinical report". The Journal of Prosthodontics 117 (2017): 205-208.

31. Unkovskiy A., et al. "Direct 3D printing of silicone facial prostheses: a preliminary experience in digital workflow". Journal of Prosthetic Dentistry 120 (2018): 303-308.

32. Revilla León M., et al. "3D metal printing - additive manufacturing technologies for frameworks of implantborne fixed dental prosthesis". European Journal of Prosthodontics and Restorative Dentistry 25 (2017): 143-147.

33. Alharbi N., et al. "Three-dimensional evaluation of marginal and internal fit of 3Dprinted interim restorations fabricated on different finish line designs". Journal of Prosthodontic Research 62 (2017): 218-226.

34. Jheon AH., et al. "Moving towards precision orthodontics: an evolving paradigm shift in the planning and delivery of customized orthodontic therapy". Orthodontics and Craniofacial Research 20.1 (2017): 106- 113.

35. Martorelli M., et al. "A comparison between customized clear and removable orthodontic appliances manufactured using RP and CNC techniques". Dental Materials 29 (2013): e1-10.

36. Reynolds M., et al. "3-d volumetric evaluation of human mandibular growth". The Open Biomedical Engineering Journal 5 (2011): 83-89.

37. Salmi M., et al. "A digital process for additive manufacturing of occlusal splints: a clinical pilot study". Journal of the Royal Society Interface 10 (2013): 20130203. 
38. Al Mortadi N., et al. "CAD/CAM/AM applications in the manufacture of dental appliances". American Journal of Orthodontics and Dentofacial Orthopedics 142 (2012): 727-733.

39. Liu Y., et al. "Protein and mRNA expressions of IL-6 and its key signaling factors under orthodontic forces in mice: an in-vivo study". American Journal of Orthodontics and Dentofacial Orthopedics 152 (2017): 654- 662.

40. Wicher J., et al. "3D Computer treatment planning in endodontics”. Journal of Dental 45 (2016): 67-72.

41. Byun C., et al. "Endodontic treatment of an anomalous anterior tooth with the aid of a 3- dimensional printed physical tooth model". Journal of Endodontics 41 (2015): 961-965.

42. Murray PE., et al. "Regenerative endodontics: a review of current status and a call for action". Journal of Endodontics 33 (2007): 377-390.

43. $\mathrm{Xu} \mathrm{HH.,} \mathrm{et} \mathrm{al.} \mathrm{"Calcium} \mathrm{phosphate} \mathrm{cements} \mathrm{for} \mathrm{bone} \mathrm{engineer-}$ ing and their biological properties". Bone Research 5 (2017): 17056.

44. Li Z., et al. "[Diagnossis and treatment of complicated anterior teeth esthetic defects by combination of whole-process digital esthetic rehabilitation with periodontic surgery]". Beijing Da Хие Хие Вао 49 (2017): 71-75.

45. Larsson L., et al. "Regenerative medicine for periodontal and periimplant diseases". Journal of Dental Research 95 (2016): 255-266.

46. Carter SSD., et al. "Additive biomanufacturing: an advanced approach for periodontal tissue regeneration". Annual Review of Biomedical Engineering 45 (2017): 12-22.

47. Li J., et al. "Evaluation of 3D- printed polycaprolactone scaffolds coated with freeze-dried platelet-rich plasma for bone regeneration". Materials 10 (2017): E831.

48. De Giglio E., et al. "Multi-compartment scaffold fabricated via 3D-printing as in vitro co-culture osteogenic model". Scientific Report 8 (2018): 15130.

49. Werz SM., et al. "3D printed surgical simulation models as educational tool by maxillofacial surgeons". European Journal of Dental Education 22 (2018): e500-e505.

50. Heym R., et al. "A new model for training in periodontal examinations using manikins". Journal of Dental Education 80 (2016): 1422-1429.
51. Lambrecht JT and Brix F. "Individual skull model fabrication for craniofacial surgery". The Cleft Palate-Craniofacial Journal 27 (1990): 382-385.

52. Kröger E., et al. "3D printed simulation models based on real patient situations for hands-on practice". European Journal of Dental Education 21 (2017): e119-e125.

53. Konvalinkova M., et al. "Using 3D digital models to establish alveolar morphotype”. Folia Morphologica 77 (2018): 536- 542.

54. Shah $\mathrm{P}$ and Chong BS. "3D imaging, 3D printing and 3D virtual planning in endodontics". Clinical Oral Investigations 22 (2018): 641-654.

\section{Assets from publication with us}

- Prompt Acknowledgement after receiving the article

- Thorough Double blinded peer review

- Rapid Publication

- Issue of Publication Certificate

- High visibility of your Published work

Website: www.actascientific.com/

Submit Article: www.actascientific.com/submission.php Email us: editor@actascientific.com

Contact us: +919182824667 